\title{
TransOral endoscopic UltraSonic Surgery (TOUSS): a preliminary report of a novel robotless alternative to TORS
}

\author{
Mario M. Fernández-Fernández • Lourdes Montes-Jovellar • \\ Pablo Luis Parente Arias • Primitivo Ortega del Alamo
}

Received: 28 August 2014/ Accepted: 29 November 2014/Published online: 16 December 2014

(C) The Author(s) 2014. This article is published with open access at Springerlink.com

\begin{abstract}
The objective of this study is to describe and evaluate the feasibility of TransOral UltraSonic Surgery (TOUSS), a new endoscopic alternative to transoral robotic surgery for approaching pharyngeal and laryngeal tumours based on ultrasonic scalpel as a resection tool. This is a prospective study on 11 consecutive patients with pharyngeal and supraglottic carcinomas between December 2013 and August 2014. All tumours were resected transorally with $35 \mathrm{~cm}$ ThunderbeatTM. Exposure was achieved using GyrusTM FK-retractor and Olympus ENDOEYE Flex $5 \mathrm{~mm}$ 2D/10 mm 3D deflecting tip video laparoscopes. We evaluated tumour staging, surgical margins, surgical time, blood transfusions, tracheostomy, enteral feeding, postoperative pain and hospital stay. The operating room setup and procedure are described. This series comprised seven early and four locally advanced carcinomas. The mean setup for TOUSS and resection time were 16 and 70.9 minutes. No major intraoperative complications were identified. The average time of nasogastric
\end{abstract}

M. M. Fernández-Fernández ( $\square)$

Department of Otolaryngology, Hospital Universitario

del Henares, Avda Marie Curie s/n, 28822 Coslada, Madrid,

Spain

e-mail: mmarcos.fernandezf@salud.madrid.org

M. M. Fernández-Fernández · L. Montes-Jovellar Otolaryngology Head \& Neck Surgery Department, MD Anderson International, c/Arturo Soria 270, 28033 Madrid, Spain

P. L. Parente Arias

Department of Otolaryngology, Hospital Universitario de A Coruña, As Xubias s/n, 15006 A Coruña, Spain

P. Ortega del Alamo

Department of Otolaryngology, Hospital Universitario de Móstoles, c/Río Júcar s/n, 28935 Mostoles, Madrid, Spain feeding tube dependence ( $n=9)$ was 13 days. Gastrostomy was performed in one patient. The average hospital stay was 14.3 days. Postoperative pain was satisfactory treated with nonsteroidal anti-inflammatory drugs. We have described TOUSS as a new feasible and intuitive procedure to approach endoscopically pharyngeal and supraglottic tumours, with good intraoperative conditions and functional outcomes.

Keywords Transoral surgery · Oropharyngeal carcinoma $\cdot$ Hypopharyngeal carcinoma $\cdot$ Supraglottic carcinoma $\cdot$ Robotic surgery $\cdot$ Thunderbeat

\section{Introduction}

Transoral robotic surgery (TORS) has demonstrated its feasibility, high rates of local control and good functional outcomes for lesions of oral cavity, oropharynx and laryngopharynx [1-4]. High definition videocameras as well as the new videoendoscopes have a critical role in its results. In fact, TORS represents a step forward in the endoscopic way to treat pharyngeal and laryngeal lesions. However, more affordable proposals are needed regarding the high costs of robotic surgery, in order to spread the endoscopic transoral approach philosophy.

Many papers have been published about the safety, utility and advantages of the ultrasonic scalpel [5]. It has been used routinely in surgical settings such as laparoscopic surgery and open abdominal and thoracic procedures in the last two decades. Specifically in head and neck surgery, it has been widely used in the last decade for open and minimally invasive thyroidectomy, and showing its potential for other open head and neck procedures like glossectomy, tonsillectomy or laryngopharyngectomy [6- 
8]. Its superior haemostasis allows clean and bloodless procedures, and the lower temperature and heat diffusion to surrounding tissue improve the safety compared with electrocautery $[5,9]$.

This paper describes a novel endoscopic approach, TransOral Ultrasonic Surgery (TOUSS), to treat laryngopharyngeal lesions, combining ultrasonic energy for cutting and coagulating, and high definition 2D-3D endoscopic imaging, in order to reach the same output of TORS.

\section{Materials and methods}

A protocol to treat human subjects with ultrasonic scalpel through endoscopic approach was designed and approved by our institutional review board. The inclusion criteria were: (1) at least 18 years old, (2) pharyngeal or laryngopharyngeal or supraglottic neoplasm with the indication for surgical excision (3) consent for transoral surgical treatment with ultrasonic scalpel. Exclusion criteria were (1) pregnancy, (2) unable to understand the surgical procedure (3) previous treatment of the laryngopharyngeal neoplasm. All patients were counselled about the alternatives to TOUSS and all of them consented to endoscopic surgical treatment of their laryngopharyngeal cancer.

\section{Laryngopharyngeal retractor}

The adequate exposition of the pharynx and the larynx was achieve through Gyrus ${ }^{\circledR}$ FK-retractor (Gyrus Medical Inc., Maple Grove, Minnesotta) as it is used for TORS.

\section{Endoscopic vision}

The endoscopic vision was achieved through both Olympus ENDOEYE Flex $5 \mathrm{~mm}$ 2D or ENDOEYE Flex $10 \mathrm{~mm} 3 \mathrm{D}$ videolaparoscopes (Olympus Medical System Corp, Tokyo, Japan). The deflectable tip allows a refinement of the surgical vision with small movements of the joysticks at the camera head, up to $100^{\circ}$ field of view in all directions. The videoendoscope and the set of laparoscopic instruments are shown in Fig. 1.

\section{Ultrasonic scalpel}

The ultrasonic scalpel Thunderbeat ${ }^{\circledR}$ (Olympus Medical System Corp, Tokyo, Japan) was used as cutting and coagulating instrument. The ultrasonic energy allows cutting and coagulating tissues simultaneously with relatively low heat and lateral thermal injury. The basic effect is similar to electrosurgery or lasers, denaturing proteins, but the mechanism of ultrasonic energy consists in transferring

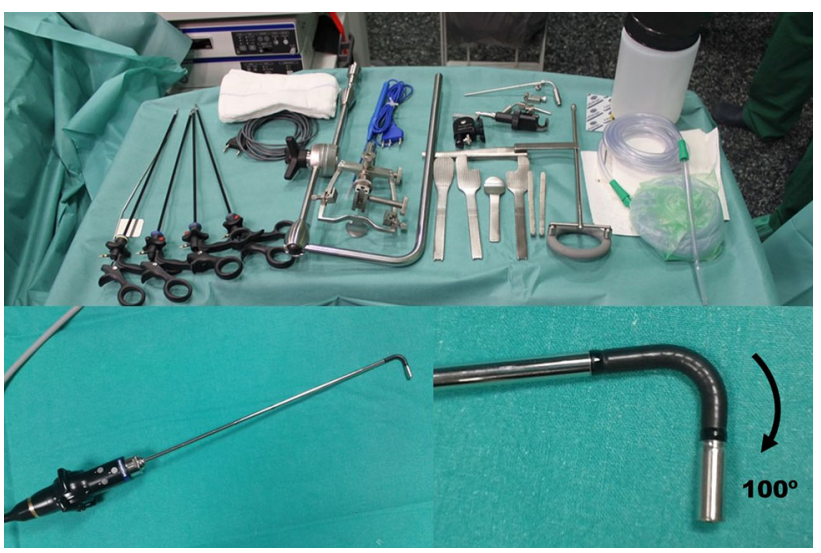

Fig. 1 Surgical instruments including the Gyrus FK-retractor, the scope holder, a set of laparoscopic forceps and scissors. At the bottom, the videolaparoscope and the deflectable tip bended at maximum are shown

to the tissue the vibrating mechanical energy at high frequency $(25-55 \mathrm{kHz})$, breaking hydrogen bonds at a low range of temperature compared with electrocautery or laser $\left(200{ }^{\circ} \mathrm{C}\right.$ maximum temperature vs $\left.400{ }^{\circ} \mathrm{C}\right)$. The cutting mechanism is achieved by a sharp blade over a distance of $100 \mu \mathrm{m}$. The precision of cutting and coagulation can be controlled by the surgeon by adjusting the power level, and lateral thermal damage is limited due to the lower working temperature. Additionally, Thunderbeat ${ }^{\circledR}$ incorporates a bipolar vessel sealing system that can be activated separately; so the possibility of additional sealing lines improves the confidence with vessels up to $7 \mathrm{~mm}$ like the lingual or upper laryngeal artery that are frequently exposed [5]. The Thunderbeat ${ }^{\circledR} 5 \mathrm{~mm} 35 \mathrm{~cm}$ shaft length allows a comfortable resection in terms of working distance.

After general anaesthesia, with the patient in supine position, the articulated arm scope holder is attached to the left side of the surgical bed and the chest support platform to the right side. The videolaparoscope is fitted into a scope holder. The monitor is place at the feet of the OR table, as well as the Thunderbeat generator (Figs. 2, 3). Tumoral resection is done under endoscopic vision, keeping the mobile jaw against the mucosa in order to reduce its damage due to direct contact with the vibrating shaft (Fig. 3). A long suction cannula is hold by the assistant to avoid the smoke overclouding the endoscopic vision when the ultrasonic device is activated (Fig. 4).

Neck dissection was performed simultaneously and prior to tumoral resection. This aspect is critical when a cervicopharyngeal communication is expected, in order to protect the carotid artery and prevent its accidental damage. Neck closure was delayed until the end of the procedure to facilitate the pharyngeal closure from outside if it was necessary. 


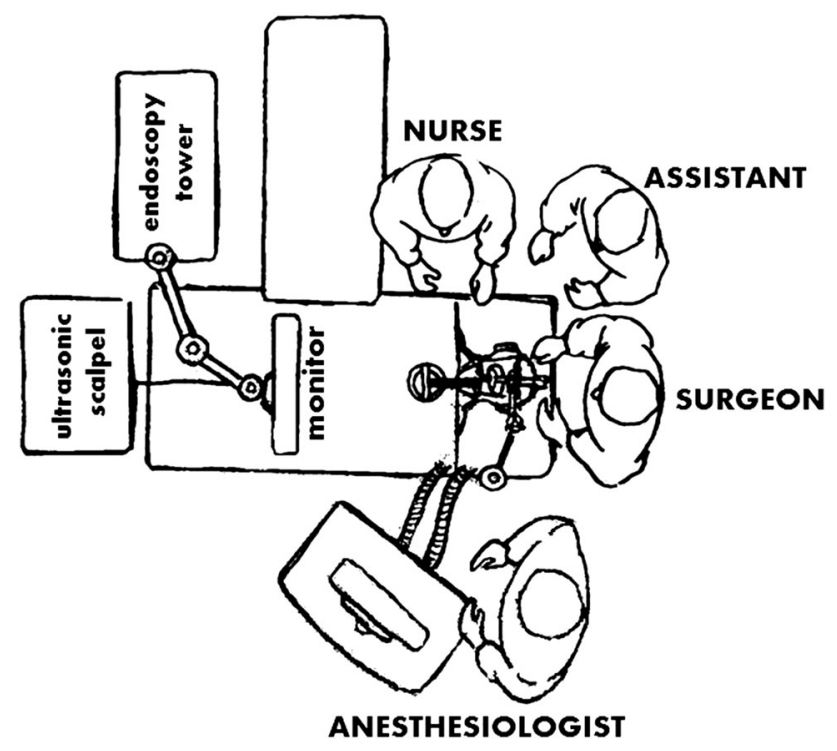

Fig. 2 Illustration showing the OR setup for TOUSS

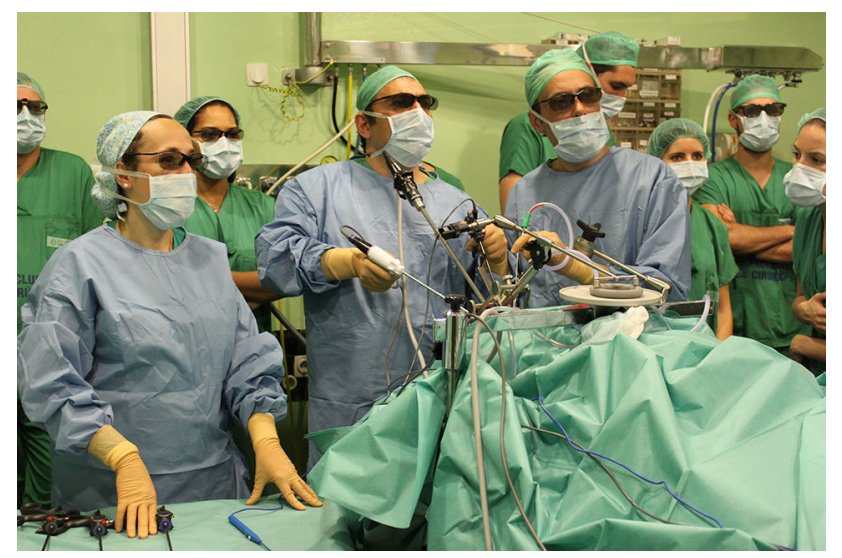

Fig. 3 TOUSS setup: the surgeon and assistant are standing up at the head of the patient. The scope holder arm is attached to the left side of the surgical bed. All the surgical team need to wear 3D glasses to watch the procedure with $3 \mathrm{D}$ endoscopy

In this study we collected data about tumour site, histology, clinical and pathological stage using AJCC criteria, TOUSS primary tumour removal operative time, TOUSS setting time, tumoral margins, tracheostomy, nasogastric or gastrostomy feeding tube, blood transfusions, hospitalization time, perioperative complications, and days of feeding tube dependence. In our institutions, carcinoma at the margin is considered a positive margin; less than $5 \mathrm{~mm}$, a close margin; and $5 \mathrm{~mm}$ or more, a clear margin. The patients were followed to assess the control of postoperative pain $(0-$ no medication needed, $1-$ mild: pain controlled with 1 nonsteroidal anti-inflammatory drug, 2moderate: pain controlled with combination of nonsteroidal anti-inflammatory drugs, and/or addition of steroids, 3severe: pain controlled with opiates; 4-uncontrollable

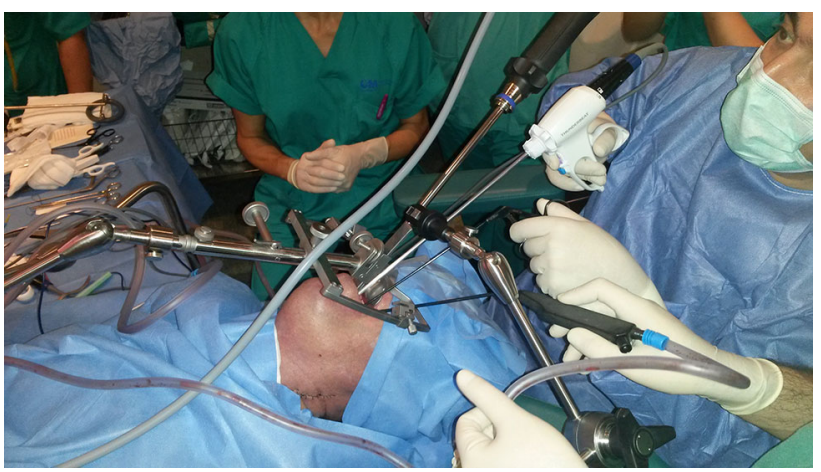

Fig. 4 Showing a closer view of TOUSS setup, the use of ultrasonic scalpel for tumoral resection and the assistant by the left side of the surgeon keeping clear the endoscopic vision with a suction cannula

pain). The data analysis was done with Microsoft Excel (Microsoft Corp, Redmond, WA).

\section{Results}

A prospective study of 11 consecutive patients who underwent TOUSS was done. All procedures were performed by the first author in four institutions. The most important data are summarized in Table 1.

Ten male and one female patients with a mean age of 60.6 years (range 46-69) were treated between December 2013 and August 2014. All patients had a history of smoking for at least 30 years (average 40.1; range 30-50) and drinking. Tumour sites were supraglottic $(n=2$; $18.2 \%)$, oropharynx $(n=6 ; 54.5 \%)$, hypopharynx $(n=2 ; 18.2 \%)$, oro-hypopharynx $(n=1 ; 9.1 \%)$. Six patients had an advanced stage III-IV carcinoma (54.6\%) and five stage I-II (45.4\%); four (36.4\%) T3-T4 and seven $(63.7 \%)$ T1-T2 carcinomas. $90.9 \%$ were treated endoscopically with TOUSS exclusively. One patient (case \#2) was treated combining an endoscopic approach (TOUSS) and a microlaryngoscopic approach using $\mathrm{CO}_{2}$ laser due to the proximity of the vocal cords to the inferior aspect of the lesion. Most patients were treated with curative intention; patient \#6 had a pulmonary metastasis (with good response to cetuximab) and the indication for surgery was set in multidisciplinary meeting to control symptoms of a locally advanced second primary tumour. Three cases $(27.3 \%)$ were second primary tumours, all of them had already undergone bilateral functional neck dissection during previous surgery, and adjuvant radiotherapy; unilateral or bilateral neck dissection was performed on the other eight patients $(72.7 \%)$. No major intraoperative complications were identified. Pharyngocervical communication was not considered a complication since it was mandatory in order to achieve a safe surgical margin. Five patients were considered at risk of pharyngocervical 


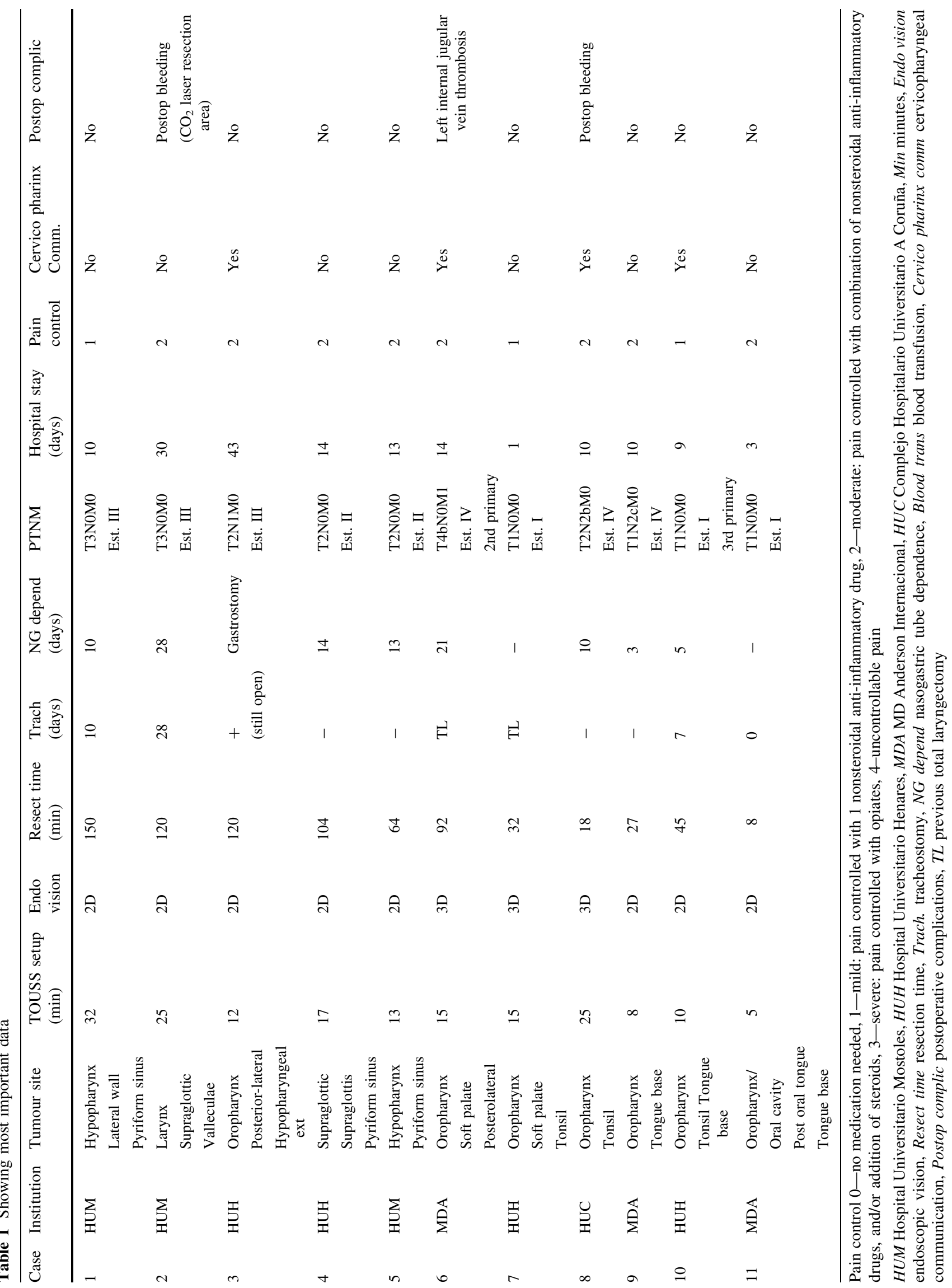


communication, due to the deep extension of the tumour and it occurred in four. All were successfully managed with direct transoral suture. An sternocleidomastoid muscle flap was used in cases \#3 and 10, to reinforce the pharyngeal suture line; one free radial forearm flap was elevated to reconstruct soft palate and lateral pharyngeal wall in case \#6 that failed in day 4 due to thrombosis of the internal jugular vein secondary to a postoperative worsening of the patient neck lymphedema (secondary to previous radiotherapy). In this patient, the cervicopharyngeal communication was successfully closed with direct suture and soft palate reconstruction was delayed. No blood transfusions were necessary for any patient at no time. TOUSS mean setup was 16 min (range 5-32). The average resection time was 70.9 min (range 8-150). The tumour was fragmented in three patients $(27.3 \%$; cases \#1, \#2 and \#6), in order to allow an adequate visualization of the inferior resection. The other eight tumours $(72.7 \%)$ were resected en bloc. The surgical margin was negative for ten patients (90.9\%), and it was uncertain for the patient \#6 treated with palliative intention. One patient (case \#3) had perineural invasion, so adjuvant radiotherapy of the primary tumour was indicated. Neck dissection was performed always prior to primary tumoral resection. 8 patients $(72.7 \%)$ underwent a neck dissection, and positive nodes were founded in three of them $(37.5 \% ; n=8)$. Postoperative complications were registered in three patients $(27.3 \%)$ : bleeding coming from the anterior commissure (case \#2) in day 1 after surgery (this area was resected with $\mathrm{CO}_{2}$ laser), an oral bleeding coming from the tonsillar area (case \#8) in day 5, both of them successfully controlled in the OR; and the internal jugular vein thrombosis referred on patient \#6. Two patients had already a total laryngectomy. Four preventive tracheostomies $(44.5 \% ; n=9)$ were performed in the other nine patients, due to the extension of the local resection (case \#3), bad pulmonary conditions (cases \#1 and 2), and difficult intubation (case \#10). Three of them were closed within 1 month (days 7, 10 and 28), and one patient (case \#4) is keeping the tracheostomy opened until the end of the adjuvant radiotherapy of the primary site. So tracheostomy was avoided in five patients. Excluding total laryngectomy patients, $100 \%$ of locally advanced tumours needed a preventive tracheostomy, but only $16.5 \%$ of the early primary lesions. Nasogastric feeding tube was inserted in nine patients $(81.8 \%)$ for an average of 13 days (range 3-28 days). When a cervicopharyngeal communication was observed, oral feeding was delayed in most patients until day 10-14. No complications related to aspiration were registered. The nasogastric feeding tube was replaced with a gastrostomy in patient \#6 before starting radiotherapy of the primary site. Patients \#7 and 11 didn't need nasogastric feeding tube. The average hospital stay was 14.3 days (range 1-43). Postoperative pain was considered mild or moderate for all patients as it was successfully treated with one intravenous nonsteroidal antiinflammatory drug in three patients $(27.3 \%)$ or a combination of two in eight $(72.7 \%)$. The need of opiate medication was not observed in any patient.

\section{Discussion}

There are an increasing number of papers reporting better functional outcomes of TORS compared with both open surgical techniques and chemoradiotherapy [10]. It is clear that transoral endoscopic approach can be a step forward and the endoscope represents a real alternative to microscope for minimally invasive approach of upper aerodigestive tract lesions. But TORS is unreachable for most of ENT departments and there is not even evidence of its costeffectiveness [11, 12]. We have designed TOUSS as a "robotless" endoscopic transoral procedure, inspired in laparoscopic setup, in order to get, at least, the same output as reported for TORS. It is mandatory to compare microscopic laser surgery with any transoral technique for laryngopharyngeal tumours. However, the endoscope means a different philosophy as it offers the possibility to enter "into the room" instead of keeping "outside the room", avoiding the need of an adequate exposition from outside the patient, as it is required for laser surgery. So our first step was to compare our results with TORS as an endoscopic procedure. Larger series of patients will demonstrate if endoscopic approach is superior to microscopic transoral approach. Shiotani has reported an experience with the same endoscopic philosophy, mainly for T1-T2 supraglottic and hypopharyngeal carcinomas, using electrocautery instruments [13]. Our design is based on ultrasonic energy as the resection tool, as the way to get a clean and safe transoral endoscopic resections of any pharyngeal and laryngeal lesion with an optimal control of the surgical margin.

Ultrasonic energy has been already used for open surgical applications in oral cavity, oropharynx, larynx and pharynx [6-8] with good oncological and functional results. Additionally TOUSS allows direct manipulation of the tissue, so the surgeon can keep the tactile input. The advantage of deflectable tip endoscopes is the easy refinement of the endoscopic visualization field with the endoscope joystick. However we cannot get conclusions about the indications for 3D endoscopic vision, but it seems that it can offer superior spatial orientation for those cases at risk of cervicopharyngeal communication.

As well as for TORS [14], indications for TOUSS could be extended to locally advanced pharyngeal tumours. In fact, four patients $(36.4 \%)$ were candidates for a mandibulotomy if an open technique were planned. The OR 
setup for TOUSS was as quick as it has been published for TORS $[4,11]$. We have observed a setting up time as low as $5 \mathrm{~min}$ after 11 cases, which is lower than the $10 \mathrm{~min}$ average time reported by Aubry et al. [12] for the robot setup after their first ten cases of TORS. An average of $70.9 \mathrm{~min}$ is already a reasonable resection time, and even a little lower than results reported by Park after 39 oropharyngeal carcinomas [15]. The reposition of the endoscope during TOUSS in order to refine the endoscopic view was identified as the most time consuming aspect of the procedure. The experience with the deflectable endoscope setup is a critical point of the learning curve.

Thunderbeat ${ }^{\circledR}$ jaws are still too bulky for working close to vocal cords when supraglottic lesions are too close to them. So until we introduce a more fine instrument, we are keeping using microscopic laser surgery only for such situation (case \#2), as it was described for TORS by other authors [16].

A fast swallowing recover has been reported for TORS $[15,17]$, with better swallowing results compared with chemoradiation $[3,4]$. Only patient \#4, with wide pharyngeal resection, keeps the tracheostomy and a gastrostomy. Return of oral feeding was possible in the other ten patients. Park had reported an average of 8.1 days of nasogastric feeding tube dependence (range 2-14) for hypopharyngeal squamous cell carcinomas [18] and 6 days for oropharyngeal carcinomas treated with TORS [14]. Genden has published an average time before starting oral intake as low as 1-3 days [19]. So this aspect is very dependent on each particular institution protocol and experience. In fact, Boudreaux et al. [20] have reported a hospital stay of 17 days. In our series, oral intake was started before discharge from hospital in all patients, and delayed to day 10-14 when a cervicopharyngeal communication had to be repaired; only patient \#10 with a small communication was considered for an earlier oral intake. The average nasogastric feeding tube dependence and hospital stay in our series was 13 and 14.3 days respectively. We avoid discharging patients until the nasogastric feeding tube could be removed and tracheostomy could be safely closed. Only one postoperative bleeding complication was attributable directly to the ultrasonic scalpel, in patient \#6, but successfully controlled in the OR. Postoperative pain was satisfactory relieved with one intravenous nonsteroidal anti-inflammatory drug or a combination of two.

In conclusion, we have described TOUSS as a new feasible, intuitive and affordable procedure to approach endoscopically pharyngeal and laryngeal tumours, even for locally advanced carcinomas, with good functional outcomes. TOUSS is a promising way to easily spread the philosophy of the endoscopic approach to the pharynx and the larynx.
Open Access This article is distributed under the terms of the Creative Commons Attribution License which permits any use, distribution, and reproduction in any medium, provided the original author(s) and the source are credited.

\section{References}

1. O'Malley BW Jr, Weinstein GS, Snyder W, Hockenstein NG (2006) Transoral robotic surgery (TORS) for base of tongue neoplasm. Laryngoscope 116(8):1465-1472

2. Weinstein GS, O'Malley BW Jr, Desai SC, Quon H (2009) Transoral robotic surgery: does the ends justify the means? Curr Opin Otolaryngol Head Neck Surg 17(2):126-131

3. Weinstein GS, O'Malley BW Jr, Cohen MA, Quon H (2010) Transoral robotic surgery for advanced oropharyngeal carcinoma. Arch Otolaryngol Head Neck Surg 136(11):1079-1085

4. Moore EJ, Olsen KD, Kasperbauer JL (2009) Transoral robotic surgery for oropharyngeal squamous cell carcinoma: a prospective study of feasibility and functional outcomes. Laryngoscope 119(11):2156-2164

5. Seehofer D, Mogl M, Boas-Knoop S, Unger J, Schirmeier A, Chopra S et al (2012) Safety and efficacy of new integrated bipolar and ultrasonic scissors compared to conventional laparoscopic 5-mm sealing and cutting instrument. Surg Endosc 26:2541-2549. doi:10.1007/s00464-012-2229-0

6. Pons Y, Gauthier J, Clément P, Conessa C (2009) Ultrasonic partial glossectomy. Head Neck Oncol. doi:10.1186/1758-3284$1-21$

7. Metternich FU, Wenzel S, Sagowski C, Jäkel T, Koch U (2002) The "Ultracision Harmonic Scalpel" ultrasound activated scalpel. Initial results in surgery of the tongue and soft palate. HNO 50(8):733-738

8. Salami A, Bavazzano M, Mora R, Dellepiane M (2008) Harmonic scalpel in pharyngolaryngectomy with radical neck dissection. J Otolaryngol Head Neck Surg 37(5):633-637

9. Kakarala K, Faquin WC, Deschler DG (2010) A comparison of histopathologic margin assessment after steel scalpel, monopolar electrosurgery, and ultrasonic scalpel glossectomy in a rat model. Laryngoscope. doi:10.1002/lary.21619

10. More YI, Tsue TT, Girod DA, Harbison J, Sykes KJ, Williams C et al (2013) Functional swallowing outcomes following transoral robotic surgery vs primary chemoradiotherapy in patients with advanced-stage oropharynx and supraglottis cancers. JAMA Otolaryngol Head Neck Surg. doi:10.1001/jamaoto.2013.1074

11. Byrd JK, Smith KJ, de Almeida JR, Albergotti WG, Davis KS, Kim SW et al (2014) Transoral robotic surgery and the unknown primary: a cost-effectiveness analysis. Otolaryngol Head Neck Surg 150(6):976-982

12. Aubry K, Yachine M, Pérez AF, Vivent M, Lerat J, Scomparin A et al (2011) Transoral robotic surgery for head and neck cancer: a series of 17 cases. Eur Ann Otorhinolaryngol Head Neck Dis 128(6):290-296. doi:10.1016/j.anorl.2011.05.002

13. Shiotani A, Tomifuji M, Araki K, Yamashita T, Saito K (2010) Videolaryngoscopic transoral en bloc resection of supraglottic and hypopharyngeal cancers using laparocopic surgical instruments. Ann Otol Rhinol Laryngol 119(4):225-232

14. Lawson G, Matar N, Remacle M, Jamart J, Bachy V (2011) Transoral robotic surgery for the management of head and neck tumours: learning curve. Eur Arch Otorhinolaryngol 268(12):1795-1801

15. Park YM, Kim WS, Byeon HK, Lee SY, Kim SH (2013) Oncological and functional outcomes of transoral robotic surgery for oropharyngeal cancer. $\mathrm{Br} \mathrm{J}$ Oral Maxillofac Surg 51(5):408-412 
16. Desai SC, Sung CK, Jang DW, Genden EM (2008) Transoral robotic surgery using a carbon dioxide flexible laser for tumors of the upper aerodigestive tract. Laryngoscope 118(12):2187-2189

17. Iseli TA, Kulbersch BD, Ideli CE, Carroll WL, Rosenthal EL, Magnuson JS (2009) Functional outcomes after transoral robotic surgery for head and neck cancer. Otolaryngol Head Neck Surg 141(2):166-171

18. Park YM, Kim WS, De Virgilio A, Lee SY, Seol JH, Kim SH (2012) Transoral robotic surgery for hypopharyngeal squamous cell carcinoma: 3-year oncologic and functional analysis. Oral Oncol 48(6):560-566
19. Genden EM, Desai S, Sung CK (2009) Transoral robotic surgery for the management of head and neck cancer: a preliminary experience. Head Neck 31(3):283-289

20. Boudreaux BA, Rosenthal EL, Magnuson JS, Newman JR, Desmond RA, Clemons L et al (2009) Robot-assisted surgery for upper aerodigestive tract neoplasms. Arch Otolaryngol Head Neck Surg 135(4):166-171 